\title{
Un portrait de la première association intellectuelle à Joliette (1856-1909)
}

\section{Luc Richard}

Volume 54, 1987

Culture et religion dans la région de Lanaudière

URI : https://id.erudit.org/iderudit/1006965ar

DOI : https://doi.org/10.7202/1006965ar

Aller au sommaire du numéro

\section{Éditeur(s)}

Les Éditions Historia Ecclesiæ Catholicæ Canadensis Inc.

ISSN

0318-6172 (imprimé)

1927-7067 (numérique)

Découvrir la revue

Citer cet article

Richard, L. (1987). Un portrait de la première association intellectuelle à Joliette (1856-1909). Sessions d'étude - Société canadienne d'histoire de l'Église

catholique, 54, 95-115. https://doi.org/10.7202/1006965ar
Résumé de l'article

L'Institut d'artisans et association de bibliothèque du village d'Industrie fut fondé en 1855 avec pour mission de combler un vide au niveau culturel, dans une région où un groupe de gens instruits se voyaient privés de livres, de journaux et d'autres moyens d'augmenter leurs connaissances. Cette association, issue à la fois de l'Institut canadien de Montréal et totalement opposée aux politiques de ce dernier, avait pour but d'encourager l'éducation et défavoriser le rayonnement de la culture en général. À titre de société littéraire, elle fut dotée d'une bibliothèque, d'une salle de lecture et d'un théâtre où se donnaient conférences, spectacles et concerts. Les membres se réunissaient régulièrement pour organiser quelques démonstrations patriotiques et religieuses, ou simplement pour discuter, sous forme de débats académiques, de questions nationales, municipales, sociales, religieuses, littéraires, etc. L'Institut ne manquait jamais d'apporter son support au clergé de la paroisse, avec lequel il entretenait d'excellentes relations. Il exerça également une grande influence sur le développement culturel, commercial et industriel de la ville de Joliette. À cause de certaines difficultés, principalement d'ordre financières, l'Institut fut contraint de fermer ses portes en 1909, après avoir brillamment servi l'élite intellectuelle de Joliette pendant plus de 53 ans.
Tous droits réservés ( Inc., 1987
Ce document est protégé par la loi sur le droit d'auteur. L'utilisation des services d'Érudit (y compris la reproduction) est assujettie à sa politique d'utilisation que vous pouvez consulter en ligne.

https://apropos.erudit.org/fr/usagers/politique-dutilisation/ 


\title{
Un portrait de la première association intellectuelle à Joliette (1856-1909)
}

\author{
Luc RICHARD \\ étudiant \\ Université de Montréal
}

\section{RÉSUMÉ}

L'Institut d'artisans et association de bibliothèque du village d'Industrie fut fondé en 1855 avec pour mission de combler un vide au niveau culturel, dans une région où un groupe de gens instruits se voyaient privés de livres, de journaux et d'autres moyens d'augmenter leurs connaissances. Cette association, issue à la fois de l'Institut canadien de Montréal et totalement opposée aux politiques de ce dernier, avait pour but d'encourager l'éducation et de favoriser le rayonnement de la culture en général. À titre de société littéraire, elle fut dotée d'une bibliothèque, d'une salle de lecture et d'un théâtre où se donnaient conférences, spectacles et concerts. Les membres se réunissaient régulièrement pour organiser quelques démonstrations patriotiques et religieuses, ou simplement pour discuter, sous forme de débats académiques, de questions nationales, municipales, sociales, religieuses, littéraires, etc. L'Institut ne manquait jamais d'apporter son support au clergé de la paroisse, avec lequel il entretenait d'excellentes relations. Il exerça également une grande influence sur le développement culturel, commercial et industriel de la ville de Joliette. À cause de certaines difficultés, principalement d'ordre financières, l'Institut fut contraint de fermer ses portes en 1909, après avoir brillamment servi l'élite intellectuelle de Joliette pendant plus de 53 ans.

\section{La naissance d'un foyer culturel}

L'industrie et la culture

De par sa vocation commerciale et industrielle, la ville de Joliette fut connue à son origine sous le nom de village d'Industrie. Les quatre ou cinq années qui suivirent le décès, en 1850, de son fondateur, Barthélemy Jo- 
liette, furent consacrées à une suite de procédures juridiques et à de longues démarches destinées à remettre un certain ordre dans les affaires de la succession. Les héritiers, en particulier son beau-frère, le docteur Peter Charles Loedel, et son neveu, Charles Barthélemy Gaspard Tarieu de Lanaudière, désiraient reprendre leur juste part, demeurée jusqu'ici concentrée entre les mains d'un seul et même homme.

Tout comme à la suite des révolutions qui font tomber les têtes couronnées, la population du village d'Industrie allait désormais se prendre en main et traavailler à la réaiisation de son autonomie jusqu'à l'incorporation municipale, obtenue en 1864.

Parallèlement au travail de colonisation entreprit par Barthélemy Joliette et poursuivi par ses successeurs, la communauté des Clercs de SaintViateur, établie à L'Industrie en 1847 , poursuivait sans relâche son œuvre d'éducation et de formation culturelle auprès des jeunes garçons de la région' ${ }^{1}$. Les habitants du village, de leur côté, auraient bien aimé se doter de structures destinées à soutenir une certaine activité culturelle au sein de la population, mais rien jusqu'à présent ne s'offrait à eux.

À titre indicatif, notons qu'en 1857 , la population du village d'Industrie s'élevait à 3500 âmes, alors que la paroisse environnante en comptait 1500 . On y retrouvait de nombreux professionnels dont cinq médecins, cinq avocats, quatre notaires et six résidents du village possédant une commission de juge de paix. Ces chiffres sont des plus très éloquents, considérant que nous sommes en présence d'une économie locale axée principalement sur le commerce du bois et de la farine ${ }^{2}$.

Nous pouvons donc affirmer, à la lumière de ces chiffres, qu'il ne manquait pas à L'Industrie de gens instruits et pourvus d'une certaine culture, mais la perspective pour ces derniers de poursuivre le développement de leurs connaissances se résumait à bien peu de chose:

À ces gens qui aimaient la lecture, qui ne manquaient pas de culture, qui désiraient augmenter leurs bagages de connaissances, qui étaient curieux des faits de l'histoire, de la littérature et des arts, il manquait quelque chose. Dans leur village, ils ne trouvaient pas la solution du problème et il leur était fort difficile de l'aller chercher ailleurs ${ }^{3}$.

1 Plusieurs études ont été consacrées à l'établissement et à l'œuvre des Clercs de StViateur à L'Industrie. Mentionnons toutefois les trois ouvrages suivants: Antoine Bernard, Les Clercs de Saint-Viateur au Canada: le premier demi-siècle, 1847-1897, Montréal, Les Clercs de Saint-Viateur, 1947, pp. 99ss; Léo-Paul Hébert, Le Québec de 1850 en lettres détachées, Québec, Ministère des Affaires culturelles, 1985, 294 p. (Collection Civilisation du Québec, 32); Pierre Robert, Vie du Père Louis Querbes, fondateur de l'institut des Clercs de Saint-Viateur (1793-1859), Bruxelles, Librairie Albert Dewit, 1922, pp. 389-431.

2 The Canada Directory for 1857-58, Montreal, John Lovell, 1857, pp. 210-211.

3 Omer Valois, «Un centre intellectuel à Joliette», L'Action populaire, 27 mai 1943, p. 1. 
En effet, vers le milieu du XIX ${ }^{\mathrm{e}}$ siècle, le village d'Industrie était relativement isolé des principaux centres de la province. Les routes conduisant à Montréal, Trois-Rivières ou Québec n'étaient guère en bon état, particulièrement au cours de l'hiver et au début du printemps, et les coûts de transport généralement élevés.

L'une des initiatives de Barthélemy Joliette fut de relier son village au fleuve Saint-Laurent par la construction d'un chemin de fer, inauguré en mai 1850. Les voyageurs pouvaient ainsi atteindre le fleuve à la hauteur du village de Lanoraie et de là monter à bord d'un bateau qui les conduisait vers Montréal ou Québec ${ }^{4}$. Malgré tout, le voyage était long, coûteux et ne s'effectuait pas toujours dans le plus grand confort.

Au cours de l'hiver, les rigueurs du climat n'incitaient pas les gens à se déplacer pour entreprendre de longs voyages. Les communications étaient donc très difficiles. Si les nouvelles provenant des grands centres prenaient souvent quelques jours avant de parvenir à L'Industrie, il fallait très souvent compter plusieurs semaines avant de recevoir les informations concernant les événements qui se déroulaient à l'étranger. La presse écrite, principal véhicule d'information, n'était certainement pas à la portée de tous. Le coût d'achat des livres et des journaux limitait la diffusion des connaissances, car bien peu de gens pouvaient se payer le luxe de les recevoir à domicile.

\section{Fondation de l'Institut}

C'est avec la ferme intention de combler un vide intellectuel que fut fondé, en 1844, l'Institut canadien de Montréal:

L'Institut canadien (...) fut bien accueilli par tout le monde... Ses objectifs (propagation de la lecture, conférences, bibliothèques, initiation à la parole publique, etc.) ne pouvaient qu'être bénéfiques aux Canadiens ${ }^{5}$.

Toutefois, les politiques adoptées par cette institution, et ce, dès ses premières années d'existence, visaient à «diffuser les idées libérales, anticléricales et même révolutionnaires et de favoriser les discussions sur la séparation de l'Église et de l'État...».6

Cette nouvelle association, dirigée en majeure partie par de jeunes intellectuels aux idées libérales, devint rapidement le symbole d'une pensée politique:

4 Jean-Claude Robert, «Un seigneur entrepreneur, Barthélemy Joliette, et la fondation du village d'Industrie (Joliette)», Revue d' histoire de l'Amérique française, 26 (1972-1973), pp. 390-392.

5 Léo-Paul Hébert, La Québec de 1850 en lettres détachées, p. 42.

6 Rosario Bilodeau, Robert Comeau, André Gosselin et al., Histoire des Canadas, Ville LaSalle, Hurtubise HMH, 1978, p. 391. 
L'Institut adopta (...) les principes et l'esprit de Voltaire, et fut dirigé en grande partie par l'influence des sociétés secrètes. En 1853-54, une seule Loge, désignée sous le nom de Francs-Frères (...), comptait environ 200 adhérents de l'Institut. Parmi eux s'inscrivirent les plus notables Libéraux ou rouges de la politique, entre autres A.-A. Dorion, Éric Dorion, Joseph Doutre, Rodolphe Laflamme, Wilfrid Laurier: les Conservateurs en étaient exclus en principe, au point de vue politique ${ }^{7}$.

L'idée mise de l'avant par ce groupe de Montréal se développa rapidement, à travers la province, au sein de communautés régionales qui, à leur tour, ressentaient le besoin de se regrouper pour y soutenir une activité culturelle jusqu'ici très peu développée:

C'est en 1844 que surgit, à Montréal, l'Institut Canadien, dont la naissance suscita à travers toute la province une floraison d'œuvres similaires. Dès, 1852, on en comptait déjà une vingtaine: à Québec, à Saint-Jean, à Chambly, à Laprairie, à Sorel, à Berthier, à Lanoraie, à Joliette, à l'Assomption, aux Trois-Rivières. Il finira (...) par y en avoir une soixantaine ${ }^{8}$.

Devant la naissance rapide, sur la scène provinciale, de ces nombreux instituts, le Parlement du Canada-Uni adopta en 1851 une loi connue sous le nom d'Acte pour pourvoir à l'incorporation et à une meilleure administration des associations de bibliothèque et des instituts d'artisans. Cette loi stipulait entre autres que:

... tout nombre de personnes, n'étant pas moins de dix, ayant souscrit ou possédant ensemble pas moins de vingt-cinq louis en argent ou en valeur pour l'usage de leur institution projetée, pourront faire signer une déclaration en double de leur intention d'établir une association de bibliothèque ou un institut d'Artisans, ou l'un et l'autre à la fois (suivant le cas), en quelqu'endroit qui sera désigné en ladite déclaration... ${ }^{9}$

L'implantation à L'Industrie d'une filiale de l'Institut canadien se réalisa grâce à l'initiative de deux figures qui ont marqué le développement de cette région: le docteur Peter Charles Loedel et le notaire Adolphe Magnan.

Peter Charles Loedel naquit à Montréal le 19 juin 1794. Après des études médicales au collège Royal de Londres, il revint au Canada et épousa, au manoir seigneurial de Lavaltrie, le 5 mars 1821, Marie Antoinette Tarieu de Lanaudière, belle-sœur de Barthélemy Joliette, futur fondateur de

7 L. Lejeune, Dictionnaire général du Canada. Ottawa, Université d'Ottawa, 1931, tome I, p. 804.

8 Omer Héroux, «Le centenaire de l'Institut Canadien», Le Devoir, 27 septembre 1948, p. 1.

9 Statuts provinciaux du Canada, 14-15 Victoria (1851), chap. LXXXVI. 
L'Industrie ${ }^{10}$. S'associant, dès 1823 , dans la majorité des entreprises de son beau-frère, il contribua à faire de leur établissement un important centre industriel. Dès la fondation de l'Institut, il fut choisi à juste titre pour occuper la présidence de l'association.

Second initiateur du projet d'un Institut d'artisans, Adolphe Magnan était natif de Barthélemy-en-Haut (Berthierville). Après des études classiques au Collège de l'Assomption de 1839 à $1843^{11}$, il s'orienta vers l'étude du notariat, fut admis à la pratique en 1850 et vint aussitôt se fixer à L'Industrie où il pratiqua sa profession jusqu'en 1896. Sachant rapidement s'illustrer au sein de sa communauté, il fonda en 1851, de concert avec le docteur Michel Séraphin Boulet, une filiale à L'Industrie de la Société Saint-Jean-Baptiste dont il fut président pendant plusieurs années ${ }^{12}$. Lors de la première assemblée de l'Institut, il fut désigné au poste de secrétairearchiviste, avant d'occuper les fonctions de président en 1861-1862, et par la suite en 1868-1869.

Le 27 octobre 1855, vingt-trois citoyens de L'Industrie, Peter Charles Loedel et Adolphe Magnan à leur tête, avaient déjà souscrit chacun la somme d'une livre et cinq chelins en vue de la mise sur pied de leur association. À cette date, une assemblée eut lieu. On procéda à la rédaction d'une déclaration légale ainsi que d'une requête destinée à obtenir une allocation auprès du gouvernement, principale source de financement de l'organisme ${ }^{13}$. Cette nouvelle association fut désignée dès lors sous le nom $d$ ' «Institut d'artisans et association de bibliothèque du village d'Industrie». La mise sur pied de l'Institut allait apporter au village d'Industrie une activité culturelle qui, aux côtés de l'œuvre des Clercs de Saint-Viateur, allait former l'embryon de cette vocation artistique qui s'y est développée icimême jusqu'à nos jours.

Le 17 décembre 1855, la déclaration fut déposée au bureau d'enregistrement du comté de Berthier et l'Institut recevait alors son existence légale.

10 Louis Richard, La famille Loedel: étude biographique sur Henry Nicholas Christopher Loedel (1754-1830), manuscrit, 1949, pp. 9, 11 et 16, Archives de la Société d'histoire de Joliette - de Lanaudière (désormais A.S.H.J.L.), dossier «Loedel (famille)».

11 Anastase Forget, Histoire du Collège de l'Assomption, Montréal, Imprimerie populaire, 1893 , p. 540.

12 Albert Gervais, Joliette illustré: numéro souvenir de ses noces d'or, 1843-1893, Joliette, Imprimerie des presses à vapeur de l'Étoile du Nord, 1893, p. 39.

13 Projet de requête adressée à la Législature, 18 février 1856, A.S.H.J.L., dossier «Institut d'artisans et association de bibliothèque du village d'Industrie». 


\section{"Le travail triomphe de tout»}

L'Institut d'artisans adopta deux devises. Pour la première, elle emprunta celle de l'Institut canadien de Montréal: «Le travail triomphe de tout» ${ }^{14}$. La seconde, «Industrie et persévérance» ${ }^{15}$, nous paraît être typiquement locale.

L'emblème de L'Institut était composé d'une locomotive et d'une charrue $^{16}$, symbolisant les deux principales activités exercées à L'Industrie et dans la région, tout faisant un rapprochement entre le passé et l'avenir.

Le sceau de l'Institut fut réalisé en 1857 par Anatole Partenais, un artiste de la région, et portait l'inscription «Institut du village d'Industrie» ${ }^{17}$.

La fête patronale de l'association fut fixée au 24 août, jour de la Saint-Barthélemy, rendant ainsi hommage au fondateur de L'Industrie, Barthélemy Joliette ${ }^{18}$.

Les réunions de l'Institut avaient lieu généralement le jeudi soir à $20 \mathrm{~h}$ du $1^{\text {er }}$ mai au $1^{\text {er }}$ octobre, et à 19 h le reste de l'année.

Les membres actifs étaient tenus de paycr "une cotisation annuelle de dix chelins, dont cinq chelins par semestre, le premier des mois de janvier et de juillet de chaque année» ${ }^{19}$. Malheureusement, tous les membres ne se conformaient pas à cette directive, ce qui plaça l'association, dès ses premières années d'activités, face à de nombreuses difficultés financières qui se poursuivirent, pour ainsi dire, jusqu' au jour de sa dissolution.

\section{Les locaux de l'Institut}

La première assemblée, convoquée pour le 19 janvier 1856, fut tenue dans la salle du marché public ${ }^{20}$, alors désigné sous le nom de marché French, du nom de son propriétaire, M. Alonzo French.

Pour la tenue de sa seconde assemblée, les membres de l'Institut se transportèrent à la résidence de leur secrétaire, le notaire Adolphe Magnan. Ce dernier accueillit sous son toit tous les membres de l'Institut, du $1^{\text {er }}$ février au 12 juin 1856. C'est à cet endroit que furent également conservés tous les volumes de la bibliothèque. On loua ensuite la maison de Louis Partenais, érigée sur un emplacement voisin du collège. On y demeura de juin 1856 à mai 1857.

14 J. L. Lafontaine, Institut-Canadien en 1855, Montréal, Sénécal \& Daniel, 1855, p. 1.

15 Ibid., p. 7.

16 Ibid..,p. 7.

17 Livre des délibérations de l'Assemblée de l'Institut d'artisans et association de bibliothèque du village d'Industrie, vol. I, pp. 25 et 70, A.S.H.J.L., registre H-41.

18 Ibid., p. 28.

19 Ibid., p. 30.

20 Ibid., p. 1. 
Dès les premières assemblées, la question de trouver un local approprié pour loger l'Institut, c'est-à-dire s'assurer d'une salle de réunions avec un endroit suffisamment grand pour y ranger et conserver livres et journaux, avec la possibilité d'y aménager une salle de lecture, fut la principale préoccupation de ses membres.

Le 27 mars 1856, un comité de cinq membres fut formé dans le but de dresser les plans et devis pour la construction de la nouvelle maison de l'Institut ${ }^{21}$. Le 25 septembre 1857, un nouveau comité chargé de l'acquisition d'une propriété pour les besoins de l'Institut présenta son rapport au président de l'assemblée, faisant état qu'à la suite d'une série de rencontres entre les membres du comité et monsieur Basile Portelance, résident de L'Industrie, ce dernier avait consenti à céder son terrain, situé sur le chemin nouvellement tracé devant l'église (aujourd'hui boulevard Manseau), pour la somme de 800 livres ancien cours, à la charge d'une rente foncière de six piastres par année ${ }^{22}$. Le comité recommanda à l'assemblée d'autoriser l'achat de cet emplacement. La proposition fut adoptée sans opposition et on donna le feu vert au début des travaux.

Le 24 juin 1858, bien que la bâtisse ne fût pas encore terminée, le curé de la paroisse, messire Antoine Manseau, présida à la cérémonie de bénédiction de l'Institut.

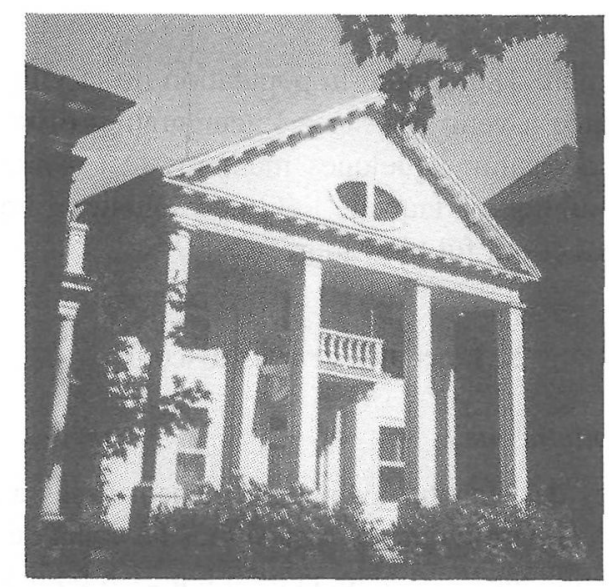

1. L'ancien édifice de l'Institut d'artisans, lequel s'élève encore aujourd' hui au 400 du boulevard Manseau à Joliette, constitue le plus ancien bâtiment de cette ville.

21 Rapport du comité de construction de l'Institut d'artisans et association de bibliothèque du village d'industrie, 17 janvier 1859, A.S.H.J.L., dossier «Institut».

22 Livre des délibérations, I, p. 75. 
Les travaux de construction du nouvel édifice, confiés aux soins de Jean Noël, un résident de Joliette, s'échelonnèrent sur près de dix ans. Au printemps 1868, le Père Joseph Michaud, c.s.v., fit don à l'Institut d'un plan destiné à compléter l'extérieur de la bâtisse, lui donnant l'apparence qu'elle conserve encore de nos jours (figure 1). Sa façade, de style néoclassique, n'est pas sans nous rappeler l'opulence de ces splendides résidences habitées autrefois par les riches planteurs du sud des États-Unis.

Les journaux de l'époque ne ménagèrent pas leurs éloges devant l'élégance du nouvel édifice de l'Institut:

Joliette doit être orgueilleuse de voir s'élever dans son sein un temple consacré à la littérature. Sans s'imposer de grands sacrifices, les membres de cette institution peuvent se flatter de posséder une bâtisse, que de plus grandes villes envieraient à Joliette. L'architecture de la façade est dans le genre dorique. C'est sans contredit le style qui convient le mieux à une institution de ce genre. Le plan en a été fourni par Messire Michaud, qui est maintenant à Rome. Les travaux ont été exécutés par M. Jean Noël, de cette ville, et font beaucoup d'honneur à cet ouvrier ${ }^{23}$.

L'édifice comprenait une salle de lecture, une salle de réunion pour lcs divers comités et une autre pour loger la bibliothèque; deux grands escaliers permettaient de communiquer, à l'étage supérieur, avec la salle de séance, annexée de deux petites chambres situées à l'arrière du fauteuil présidentiel et pouvant servir de loges lors des représentations dramatiques $^{24}$.

Le 19 novembre 1868 , toute la population de la ville de Joliette fut cordialement invitée à venir assister à l'inauguration officielle du nouvel édifice. À cette occasion, «quelques membres (...) avaient préparé une séance littéraire publique à laquelle l'élite de Joliette et bon nombre d'étrangers assistaient et qui fut bien appréciée» ${ }^{25}$.

\section{L'ère des grands discours}

\section{Regard sur la bibliothèque}

Les membres de l'Institut avaient facilement accès à leur bibliothèque. La salle de lecture ouvrait ses portes, tous les jours de la semaine, de $6 \mathrm{~h}$ à $18 \mathrm{~h}$, du $1^{\text {er }}$ mai au $1^{\text {er }}$ octobre, et de $8 \mathrm{~h}$ à $21 \mathrm{~h}$ pour le reste de l'année, à l'exception des heures où se tenaient les offices dominicaux et lors des jours de fête d'obligation.

23 La Gazette de Joliette, 26 octobre 1868, p. 2.

24 Rapport du comité de construction, 17 janvier 1859.

25 «Une séance littéraire», La Gazette de Joliette, 23 novembre 1868, p. 2. 
Sans négliger les vastes connaissances et les grandes qualités d'esprit de ses membres, la bibliothèque de l'Institut représentait, il va s'en dire, la principale richesse, tant matérielle qu'intellectuelle, de cette association. Peu fortuné, l'Institut favorisait plus particulièrement les abonnements aux différents journaux et laissait à la discrétion de ses membres le soin de garnir les rayons de la bibliothèque par les dons qu'on acceptait de lui faire.

Le 27 février 1856, le secrétaire correspondant de l'Institut Canadien de Montréal, Louis A. Jetté, faisait savoir aux membres de l'Institut du village d'Industrie qu'on allait enrichir sous peu leur bibliothèque: «J'ai l'honneur de vous annoncer que l'Institut Canadien, ayant accueilli favorablement votre demande, a décidé à sa séance du 14 courant, de faire don à votre société de 57 volumes de divers ouvrages» ${ }^{26}$.

L'un des premiers devoirs du bibliothécaire consistait à «faire un choix de tous les livres qui peuvent être mis en circulation et en éliminer tous les livres immoraux ou obsènes qui pourront s'y trouven ${ }^{27}$.

Outre les œuvres littéraires mises à la disposition de ses membres, la bibliothèque souscrivit à de nombreux journaux, tant locaux qu'étrangers, lesquels formaient la principale source d'information où les membres de l'Institut pouvaient puiser une matière abondante servant à alimenter leurs débats. Parmi les titres reçus mentionnons deux journaux étrangers: $L e$ Courrier des États-Unis et L'Illustration de Paris; quelques grands journaux de la province: La Nation, La Tribune, La Presse, The Gazette, Le Courrier du Canada, La Minerve, Le Canadien, L'Opinion publique, etc.; et, bien entendu, nos journaux locaux: L'Écho des campagnes (Berthier), La Gazette de Joliette, et L'Industrie.

La majorité des membres (certains noms de consonance anglophone ne sauraient trahir leur origine) employaient couramment et avec aisance la langue anglaise. Dans la salle de lecture, nos journaux francophones côtoyaient des publications telles que The Gazette, le Montreal Herald, le Canadien Illustrated News et le Daily News de Montréal. D'ailleurs, l'article 19 de l'appendice à la constitution de l'Institut stipulait que «toute motion ou autre procédé écrit de l'Institut doit être fait en langue française, mais il sera permis de parler sur toute question en langue anglaise....»28.

Au cours de ses années d'activités, l'Institut offrit près d'une quarantaine de journaux à ses membres. Lorsqu'un d'entre eux désirait lire un journal auquel n'était pas abonné l'Institut, il en proposait l'abonnement au

26 Louis A. Jetté à François Benjamin Godin, 27 février 1856, A.S.H.J.L., dossier «Institut».

27 Livre des délibérations, I, p. 61.

28 Ibid., p. 28. 
cours d'une assemblée et la demande était habituellement acceptée. À l'opposé, lorsqu'un journal déplaisait à un membre, ce dernier ne se gênait guère pour demander l'annulation de l'abonnement, attitude qui ne plaisait pas toujours à ses confrères. Cette situation donnait souvent naissance à des discussions orageuses auxquelles le président devait mettre fin par procédure démocratique et un vote mettait fin au débat.

Le 7 février 1878, la bibliothèque de l'Institut enrichissait ses rayons en faisant l'acquisition de l'imposante Encyclopédie du XIX ${ }^{\mathrm{e}}$ siècle (4e édition) en 70 volumines ${ }^{20}$.

Plusieurs années après la fermeture de l'Institut, un de ses anciens membres, le notaire Samuel Alfred Lavallée, alors âgé de 78 ans, se souvenait encore avec précision des ouvrages dont était composée la bibliothèque:

... en 1892, la bibliothèque était encore remplie d'ouvrages portant les exlibris de la «maison-mère» de Montréal. Ces ouvrages étaient presque tous à l'Index. (...) Les rayons contenaient encore des écrits d'une saveur fortement maçonnique, telles les Oeuvres complètes de Chamfort, Les égarements de la raison, du comte de Valmont, un produit des Salons de l'époque, Les mémoires de Ninon de Lenclos. Tous ces livres portaient le sigle de l'Institut canadien de Montréal. Ils sont demeurés dans la bibliothèque jusqu'à la fin... ${ }^{30}$

\section{La question du jour}

L'une des principales activités inscrite à l'ordre du jour des assemblées de l'Institut d'artisans - et certainement la préférée de ses membres - consistait à soumettre une question sur un sujet d'intérêt local, national ou autre, destinée à y être discuter lors d'une réunion ultérieure. Certains membres prenaient alors position, préparant soigneusement leurs arguments avec la ferme intention de défendre leur position lors d'un débat où tous aimaient faire valoir à l'occasion leur talent d'orateur.

Les sujets choisis furent des plus variés; nous en retiendrons cependant quelques-uns parmi les plus significatifs. On y discutait notamment de politique nationale: «L'annexion du Canada aux États-Unis serait-elle préjudiciable à notre nationalité?» ${ }^{31}$ «Québec est-il préférable à Montréal pour le siège du gouvernement?» ${ }^{32}$ "Serait-il avantageux d'abolir l'indemnité parlementaire au Canada et dans la province de Québec?»" «Est-il plus avantageux pour le peuple canadien de maintenir le lien colonial (avec

29 Ibid., p. 355.

30 Marcel Hamel, «Le notaire S.-Alfred Lavallée remue les cendres de l'Institut», $L^{\prime} E$ toile du Nord, 9 septembre 1948, p. 10.

31 Livre des délibérations, I, p. 42.

32 Ibid., p. 220.

33 Ibid., p. 14. 
l'Angleterre), ou bien de déclarer son indépendance, ou bien encore de s'annexer aux États-Unis?» ${ }^{34}$

Les questions d'intérêt régional occupaient également une large part des débats: «Serait-il plus avantageux pour l'agrandissement du village d'Industrie que les édifices d'un palais de justice, prison, etc., soient érigés sur le côte nord-est de la rivière l'Assomption, dans les limites du village, ou sur le côté nord-ouest?» ${ }^{35}$ «Serait-il avantageux pour le village (d'Industrie) de se faire incorporer en ville? $»^{36}$ «La ville de Joliette progresserait-elle plus par l'établissement de manufactures que par l'établissement d'une voie ferrée traversant le district de Joliette et se rattachant au chemin de fer de colonisation du nord? $»^{37}$

Mais il va s'en dire que les membres privilégiaient tout particulièrement les questions d'ordre social, là où le talent d'orateur et la finesse d'esprit pouvaient être réellement mis à l'épreuve. Il n'y a qu'à jeter un coup d'œil sur les questions suivantes pour le constater: «La religion a-telle plus contribué au progrès de la civilisation des peuples que le commerce?» ${ }^{38}$ «De l'avocat, du médecin et du notaire, quel est celui qui rend plus de service à la société? ${ }^{39}$ «La gloire des lettres est-elle plus belle que celle des armes? $\rangle^{40}$ «L'usage du tabac est-il préjudiciable à la santé» ${ }^{41}$ «L'usage immodéré des liqueurs enivrantes est-il plus dommageable à l'homme généralement que l'usage immodéré du tabac?»42

Ces quelques questions ne sont qu'un exemple des nombreux sujets inscrits à l'ordre du jour des assemblées. Certains de ces débats opposèrent, pendant de longues soirées, les plus fins orateurs, au grand plaisir de tous les membres de l'assemblée.

\section{Discours et débats}

Le débat le plus houleux que connut l'Institut prit naissance lors de la séance du 23 avril 1868. Le notaire Placide Renaud proposa alors de renvoyer le journal Le Pays. Les politiques soutenues par cet organe de presse allaient à l'encontre des opinions de certains membres de l'Institut:

Fondé par J.-A. Plinguet et Édouard-Raymond Fabre, le Pays remplaçait l'Avenir comme organe du parti rouge. D'un ton plus modéré que l'Avenir, il

\footnotetext{
34 Ibid., p. 241.

35 Ibid., p. 45.

36 Ibid., p. 83.

37 Ibid., p. 233.

38 Ibid., p. 19.

39 Ibid., p. 258.

40 Ibid., p. 163.

41 Ibid., p. 129.

42 Ibid., p. 239.
} 
n'en continuait pas moins la lutte pour le triomphe de l'idéal démocratique. (...)

Le Pays soutint, en 1867, la campagne de Lanctôt contre la Confédération et prôna, en 1870, avec Galt, l'annexion aux États-Unis. ${ }^{43}$

L'opposition fut très forte et la question ne tarda pas à diviser l'assemblée. Rapidement, le ton s'éleva dans les deux camps. Croyant ne jamais réussir à trouver la voie du compromis, on suggéra alors de confier le règlement de cette affaire au Père Pascal Drogue Lajoie, curé de la paroisse, et d'accepter intégralement sa sentence. Plusieurs membres protestèrent vivement contre une telle solution en alléguant que cette affairc devaiit se lí́gler entre les murs de l'Institut, sans devoir recourir à l'autorité religieuse. Cette proposition fut acceptée et la discussion reprit de plus belle. Le débat se poursuivit jusqu'aux petites heures du matin, alors que la majorité décida de renvoyer ce journal, par un vote de vingt-et-une voix contre neuf ${ }^{44}$.

Lorsqu'il apprit la décision rendue par l'Institut de Joliette, Le Pays en fut vivement offensé et attaqua avec violence l'intégrité des membres de l'association. Le joumal les traita de tous les noms, les accusant de posséder une étroitesse d'esprit comme on en rencontre peu. Cet échange provoqua une gucrre de plumes à laquelle prirent part plusieurs journalistes, dont Joseph Martel, avocat de Joliette, reconnu à l'époque pour ses qualités d'écrivain et d'orateur, un homme «à la répartie vive, à la farce plaisante, au calembour plein de finesse et d'astuce $»^{45}$. Cet événement, sans grande importance au départ, déclencha une vive polémique à laquelle participa un jeune journaliste du nom de Joseph-Israël Tarte, alors à peine âgé d'une vingtaine d'années.

Quelques temps après cet incident, l'Institut réserva le même sort au journal Le Nouveau-Monde. Ce dernier s'opposait au projet de construction d'un chemin de fer sur la rive nord du Saint-Laurent, projet appuyé à l'unanimité par les membres de l'Institut et par lequel la ville de Joliette comptait retirer de nombreux avantages.

\section{Théâtre et musique}

L'Institut aimait recevoir à l'occasion la troupe des amateurs de la ville de Joliette, de même que certaines formations musicales ou théâtrales de la région. Celles-ci organisaient régulièrement des soirées dramatiques au profit de certaines œuvres de charité ou simplement dans le but d'offrir un agréable divertissement: «C'est là que de jeunes talents ont ouvert leurs

43 André Beaulieu et Jean Hamelin, Les journaux du Québec de 1764 à 1964, Québec, Les Presses de l'Université Laval, 1965, p. 137.

44 Livre des délibérations, I, pp. 182-185.

45 J. Édouard Gervais, Joliette, 1864-1964, Joliette, Imprimerie nationale, 1964, p. 132. 
ailes à la recherche de la gloire et que des artistes distingués ont donné au public des soirées intéressantes... $\rangle^{46}$.

Il arriva même que la population puisse venir y applaudir les performances de quelques artistes de réputation internationale (figure 2).

\section{Religion et culture}

Au départ, l'Institut d'artisans du village d'Industrie ne fut nullement organisé avec l'intention de défendre l'Église et la foi; là n'était pas son but. Cependant, tout porte à croire qu'il eut un rôle positif à jouer vis-à-vis cette question. À L'Industrie, religion et culture entretenaient d'ailleurs des liens très étroits.

Malgré la guerre que s'étaient déclarés l'Institut canadien et l'évêque de Montréal, Monseigneur Ignace Bourget, querelle devant mener à la célèbre «affaire Guibord», jamais au cours de l'histoire de l'Institut d'artisans, il n'y eut le moindre signe d'incompatibilité entre l'association et les autorités religieuses de la région. Au contraire, nous pourrions, dans une certaine mesure, parler de collaboration étroite entre les deux. Il fut reconnu, et nous n'avons guère raison d'en douter, que les membres de l'Institut étaient tous fervents catholiques et pratiquants. De plus, il était clairement établi dans les règlements de l'association que les portes de l'Institut devaient demeurer fermées pendant la tenue des offices religieux du dimanche et lors des jours de fête d'obligation.

Au cours de l'assemblée du 18 avril 1856, le curé de la paroisse, le grand vicaire Antoine Manseau, et le Père Pascal Drogue Lajoie, alors directeur du Collège Joliette, les deux principales personnalités religieuses de l'époque à L'Industrie, furent admis à titre de membres honoraires de l'Institut ${ }^{47}$. En plus de Messieurs Manseau et Lajoie, on remarque que certains professeurs du Collège Joliette venaient assister à l'occasion aux assemblées régulières.

Le 10 mai 1868, à la veille du départ pour Rome du Père Joseph Michaud, c.s.v., les membres de l'Institut rédigèrent l'adresse suivante:

À Messire Jos. Michaud, ptre, professeur de physique au Collège Joliette.

Monsieur.

Je viens au nom de l'Institut de Joliette vous présenter, à la veille de votre départ pour Rome, nos sentiments de reconnaissance pour les services que vous avez su nous rendre, et en même temps nos souhaits sincères et chaleureux qui vous accompagneront dans votre voyage. Nous sommes persuadés que si nos vœux sont exaucés, votre voyage sera prospère et votre retour dans notre ville,

46 Omer Valois, «Un centre intellectuel à Joliette», L'Action populaire, 10 juin 1943 , p. 5.

47 Livre des délibérations, I, p. 39. 
Mercredi, 12 Août 1868.

\section{CONCERT COMIQUE.}

Soirée d'Adieu.

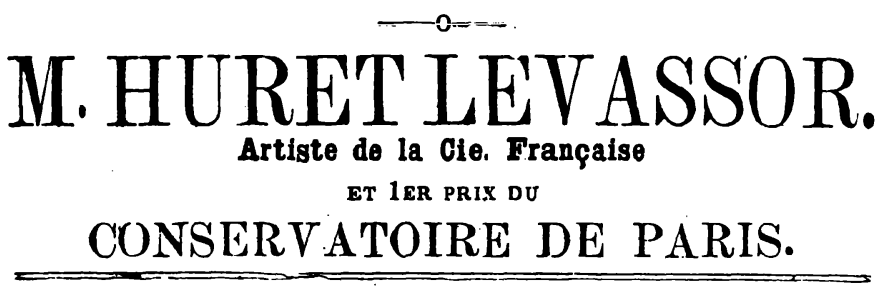

RRR PARTRE.

1-Un maringe excentrique, Scène comiq̣ue (redemandé)

2-Tout ce que l'ur: pent faire avec un sou

3-'Tyrolienue, Morceall italien

4-Le Beau Nicolus, chansonnette

5-Les primeurs de la vie, Romance

6-L'angla is mélunane, Chansomett comique

7-Pile ou lace, grande hésitation en 4 couplets

Auteurs.

Parizot.

H. Levassur

Villi.

Berger.

Clapissan.

H. Levassor,

H. Levassor.

Intermède de 10 minutes.

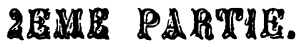

1-Le Waggon littéraire, grande Scène

2-Pretty litlle Sarah, english comic Sung

3-La plainte du'mousse, mélodie

4-Les plaideurs, grande Scène d'imitation

5-Les poëtes de la France, devant Jupiter

6-Le chien de la vellve Lenglumé, (Redernandé)

7- L'anglais très gai, grınde Scène comique

\section{A UTEurs.}

H. Levassor, rimith.

'Travio.

H. Levassor.

Anonyme.

H. Lev vassur

H. Levilssur.

A la demande générale, M. H. Levassor a bien voulu se décider à redonner pour la dernière $f(i s$ sa grande parodie da grand opera de Lncie.

$$
\text { "Lucie de la Mère horeau." }
$$

\section{A muission : 25 cts, Enfants : mortie prix.}

Les portes seront ouvertes à 7 heures. Le Concert conımencera à 8 heures I'. M.

N B.-On pourra se prucurer des Billets à l'avance-chez M. Lprohou-Bureulu du télégra lhe el ì l'hôtel Butrdon.

2. Affiche publicitaire annonçant la tenue d'une grande soirée de concert à la salle de l'Institut, le 12 août 1868. 
prompt et heureux. Nous ne doutons pas que vous saurez utiliser le séjour que vous ferez dans la patrie du Raphaël et de Michael-Ange, et que vos talents y acquéreront un brillant éclat qui rejaillira sur votre patrie et vos concitoyens. Assurez notre Saint Père de notre soumission à l'Église, de notre adhésion aux principes qu'il défend et de l'aversion que nous avons pour les doctrines révolutionnaires qui se déchaînent contre lui. Permettez-nous de vous le répéter, que votre voyage soit heureux; nos vœux vous accompagnent.

Adolphe Magnan, président. ${ }^{48}$

L'assemblée se transporta ensuite chez le Père Michaud. Le président lui fit lecture de l'adresse et lui offrit par la même occasion un présent de la part de l'Institut; une excellente preuve des sentiments qui animaient l'esprit de ses membres.

Le $1^{\text {er }}$ février 1878, messieurs Jean-Baptiste Chapdelaine et Eusèbe Asselin proposèrent d'amender la constitution de l'association afin d'inclure une condition à l'article 3 de l'appendice, dans le but de s'assurer que toute personne désirant à l'avenir devenir membre de l'Institut soit obligatoirement de confession catholique. Un débat fut ouvert et, après avoir soumis cette question au vote de l'assemblée, elle fut adoptée par une forte majorité de 17 voix contre 2 . Dorénavant, l'article 3 de la constitution allait se lire comme suit:

Peut êtré membre correspondant, toute personne demeurant hors du village d'Industrie et désirant favoriser l'Institut de communications littéraires ou scientifiques; et peut être membre actif, toute personne de quelque origine qu'elle soit, pourvu qu' elle appartienne à la religion catholique romaine. ${ }^{49}$

Le 17 décembre 1901, l'abbé Théophile-Stanislas Provost fut invité à venir prononcer une conférence devant les membres de l'Institut. Il leur fit alors la lecture d'un travail très élaboré qu'il venait de compléter sur la vie et l'œuvre de Monseigneur Jean-Jacques Lartigue, premier évêque de Montréal, et «qui [fut] écouté avec plaisir et attention» ${ }^{50}$

\section{Loyauté et patriotisme}

Animés de sentiments loyalistes, les membres de l'Institut eurent l'occasion d'exprimer à plusieurs reprises leur attachement à la couronne britannique, en particulier lors du décès de leur souveraine, la reine Victoria. Au cours de l'assemblée du 24 janvier 1901, ils adoptèrent une série de résolutions dont la lecture fut donnée par leur président, J. Mathias Tellier:

L'empire britannique est aujourd'hui dans le deuil. Le décès de l'Auguste Souveraine qui depuis plus de 63 ans a présidé avec tant de gloire aux destinées

48 Ibid., pp. 191-192.

49 Ibid., pp. 298-299.

50 Livre des délibérations de l'Assemblée de l'Institut d'artisans et association de bibliothèque du village d'Industrie, vol. II, p. 128, A.S.H.J.L., registre H-42. 
de ce vaste empire, afflige profondément le cœur de tous ses sujets. Jamais en effet, souveraine ne fut plus aimée, plus respectée, ni plus digne de l'être. Victoria I a donnée l'exemple de toutes les vertues comme reine, épouse et mère. Le bonheur de ses sujets a toujours été la plus grande de ses préoccupations. (...) Les membres de l'Institut, en particulier, aiment aussi à se rappeler que c'est durant le même règne que leur institution a pris naissance, a grandi et s'est développée en toute liberté. Aux sentiments de regrets que nous cause la perte de notre Auguste Souveraine, nous joignons nos sentiments de respectueuse soumission et nos meilleurs vœux pour son très illustre fils, notre nouveau roi, Édouard VII. ${ }^{51}$

Patriotes, les membres de l'Institut ne manquaient jamais de célébrer la Saint-Jean-Baptiste. On se réunissait devant l'édifice de l'Institut pour ensuite se rendre à l'église où une messe était célébrée. À la sortie du saint office, un défilé était organisé dans les rues de la ville après quoi, revenant à leur point de départ, les uns s'arrêtaient pour y entendre les autres prononcer quelques discours de circonstance.

\section{Une association en crise}

Très tôt, l'enthousiasme des premières années fit place à un certain désintéressement des membres et plusieurs d'entre eux négligèrent $d$ 'assister aux assemblées régulières. Pour contrer ce problème, on songe à faire payer une amende aux membres qui, pour une raison jugée non valable, s'absentaient des réunions. On ne donna toutefois jamais suite à cette proposition.

\section{Difficultés financières}

Dès ses premières années d'existence, l'Institut fut touché par une crise financière. Pour faire face à cette situation, on recommanda, le $1^{\text {er }}$ mars 1860 , de renvoyer tous les journaux auxquels l'Institut avait souscrit un abonnement, mais cette solution, jugée trop radicale, fut rejetée par la majorité des membres, alléguant qu'une telle décision porterait atteinte au caractère littéraire que s'était donné cette institution.

L'institut d'artisans vécu toujours dans une situation financière extrêmement précaire. Plusieurs membres refusaient de payer leur cotisation et l'on n'hésitait pas alors à rayer leur nom de la liste et à saisir la part de capital qu'ils avaient souscrit lors de leur admission. À l'occasion, l'Institut fut contraint de sévir contre certains de ses membres, allant des menaces jusqu'à l'obligation d'intenter contre eux des poursuites en justice dans le but de réclamer ce qui lui était dû.

51 Ibid., pp. 124-125. 


\section{Nouvelles vocations}

Divers moyens furent mis de l'avant pour résoudre les difficultés financières auxquelles l'Institut fut toujours confrontées. Une première source de revenus additionnels consistait à louer les salles à divers organismes locaux, voire à certains particuliers qui désiraient y exercer leurs activités. Le 16 mars 1865 , on apprend que des leçons de fleuret sont offertes à la population dans la grande salle de l'institut ${ }^{52}$. Dans le même ordre d'idée, on fit la location, le 18 mars 1869 , de la grande salle du second étage, habituellement réservée aux représentations théâtrales, à monsieur Moïse Ritchot qui désirait y installer un vélocipède ${ }^{53}$ ! Les citoyens de Joliette étaient alors invités à venir se familiariser avec ce nouveau moyen de locomotion.

Le 22 octobre 1874, un dénommé Trudeau, président de la Compagnie de gymnastique de la ville de Joliette, avisa l'Institut qu'à la prochaine assemblée de ses membres, une demande serait présentée de sa part à l'effet d'utiliser la salle de théâtre pour les exercices de gymnastique. Hésitant à louer leurs spacieux locaux pour un tel usage, quelques membres de l'Institut formèrent un comité chargé d'enquêter sur les dommages qui pourraient éventuellement être causés à la structure de la salle. On alla consulter le Père Joseph Michaud qui, après avoir examiné attentivement les lieux, donna son approbation, et un bail fut passé avec Trudeau, lui permettant d'utiliser la salle les lundi, mercredi et vendredi, de $19 \mathrm{~h}$ à $21 \mathrm{~h}$ $30^{54}$.

\section{Un second souffle}

L'année 1885 marqua en quelque sorte la fin d'une époque, car c'est à ce moment que se produisit un tournant décisif, rendant un verdict final sur l'avenir de l'association.

À partir de 1886, on assista, après avoir vécu une période difficile, à une reprise des activités de l'Institut. On procéda à l'agrandissement de l'édifice, ainsi qu'à une nouvelle orientation des activités de l'organisme. On introduisit, dans un premier temps, une panoplie de jeux dans les différentes salles de l'Institut (jeux de dame, de billard, de cartes, tennis de table et autres). D'après l'histogramme de la fréquence annuelle des assemblées de l'Institut, il semble que cette époque ait été marquée par une reprise des activités de l'association, mais uniquement pour une brève période. Ces changements attirèrent une nouvelle et nombreuse clientèle au

52 Livre des délibérations, I, p. 134.

53 Ibid., p. 227.

54 Ibid., pp. 238-242. 
sein de l'association dont on vit la liste des membres s'allonger rapidement, ce qui n'était pas nécessairement un signe de progrès.

Il serait difficile de chiffrer avec précision le nombre de ceux qui furent admis comme membres de l'Institut d'artisans au cours de ses cinquante-trois années d'existence. Toutefois, un estimé sommaire réalisé après lecture des procès-verbaux de chacune des assemblées nous permet d'évaluer ce nombre à près de trois cent cinquante.

Il est à signaler toutefois que ce second souffle, apporté par un changement de vocation, ne reçut guère l'assentiment de tous et ne réussit pas à plaire à certains membres, en particulier aux plus anciens qui avaient vécu les premières années d'activités et les heures de gloire de leur société. $\mathrm{Si}$, d'un côté, le nombre des membres s'élevait, de l'autre côté, les démissions se firent plus nombreuses que jamais. Cette institution qui se voulait être au départ un foyer culturel, une association à caractère littéraire, ressemblait maintenant beaucoup plus à un club récréatif.

Le 9 février 1905, l'avocat Ernest Hébert proposa d'amender les règlements dans le but de permettre à l'Institut de demeurer ouvert jusqu'à minuit tous les soirs et durant les vêpres, les dimanches et les jours de fête d'obligation. La motion fut aussitôt rejetée ${ }^{55}$.

Le malaise s'accentua au sein de l'assemblée et plusieurs de ses membres, voyant l'avenir d'un mauvais œil, purent dès lors prédire que le prochain chapitre allait probablement être le dernier.

\section{Dissolution}

Au mois de décembre 1907, devant les nombreuses difficultés auxquelles devait faire face l'Institut d'artisans, on s'interrogea sérieusement sur l'avenir de l'association. Dans un premier temps, on constatait le besoin urgent des réparations qui devaient être apportées à l'édifice de la rue Manseau. Face à une situation financière toujours très précaire, on songea à faire passer la cotisation annuelle de $4 \$$ à $10 \$$ ou $12 \$$. L'assemblée des membres jugea cette solution inacceptable ${ }^{56}$.

55 Livre des délibérations, II, pp. 165 et 168.

56 Ibid., p. 182. 
Lors de l'assemblée du 3 mai 1909, il fut décidé que l'Institut devait malheureusement fermer ses portes dès le mois suivant. L'édifice de l'association fut vendu à dame Amanda Dauphin, épouse de J. P. Léon Ducharme, avocat et actuel président de l'Institut, pour la somme de 2500 \$ comptant. Tout le mobilier et les diverses installations qui s'y trouvaient allaient être vendu sous peu par encan public. Tout porte à croire que le même sort fut réservé aux nombreux ouvrages que renfermait la bibliothèque. Le 11 juin 1909, les membres de l'Institut furent appelés à se réunir en assemblée extraordinaire, au bureau du notaire Samuel Alfred Lavallée, pour procéder à la rédaction de l'acte de dissolution:

... il a été passé et adopté une résolution décrétant la dissolution de cette association et l'attribution à chacune des personnes faisant partie du dit Institut à la date du trois mai mil neuf cent neuf, ou à leurs représentants, des argents composant l'actif de l'association....

Suivaient les vingt-et-une signatures de ceux qui venaient d'avoir la triste tâche d'ensevelir une tradition culturelle vieille de plus d'un demisiècle.

\section{Conclusion}

Au cours de la seconde moitié du XIX ${ }^{\mathrm{e}}$ siècle, le développement de la vie culturelle à Joliette reposa principalement sur la responsabilité de deux groupes d'individus: un premier, à vocation religieuse, ayant à sa tête la communauté des Clercs de Saint-Viateur; et un second, formé de laïcs, qui avait pour but de regrouper l'élite intellectuelle de la ville et dont nous venons de tracer le portrait. En 1943, Monseigneur Omer Valois, alors secrétaire de la Société historique de Joliette, s'exprimait en ces termes:

Aussi avec combien de raison pouvons-nous affirmer, avec nos historiens et avec la tradition, que Joliette compta toujours dans son sein une élite intellectuelle, un groupe d'hommes et de femmes cultivés, distingués, curieux des choses de l'esprit, s'intéressant aux lettres, à la musique, à la peinture, à la mécanique, aux progrès de la science, à l'éloquence et à tout ce qui fait la gloire d'une époque ou même d'une civilisation. ${ }^{58}$

57 Ibid., p. 194.

58 Omer Valois, «Un centre intellectuel à Joliette», L'Action Populaire, 27 mai 1943, p. 1. 
Et cette tradition demeure toujours présente au cœur d'une ville qui ne cesse de récolter, aujourd'hui, les fruits qui furent autrefois semés et cultivés avec patience et acharnement derrière les murs de l'Institut d'artisans. 


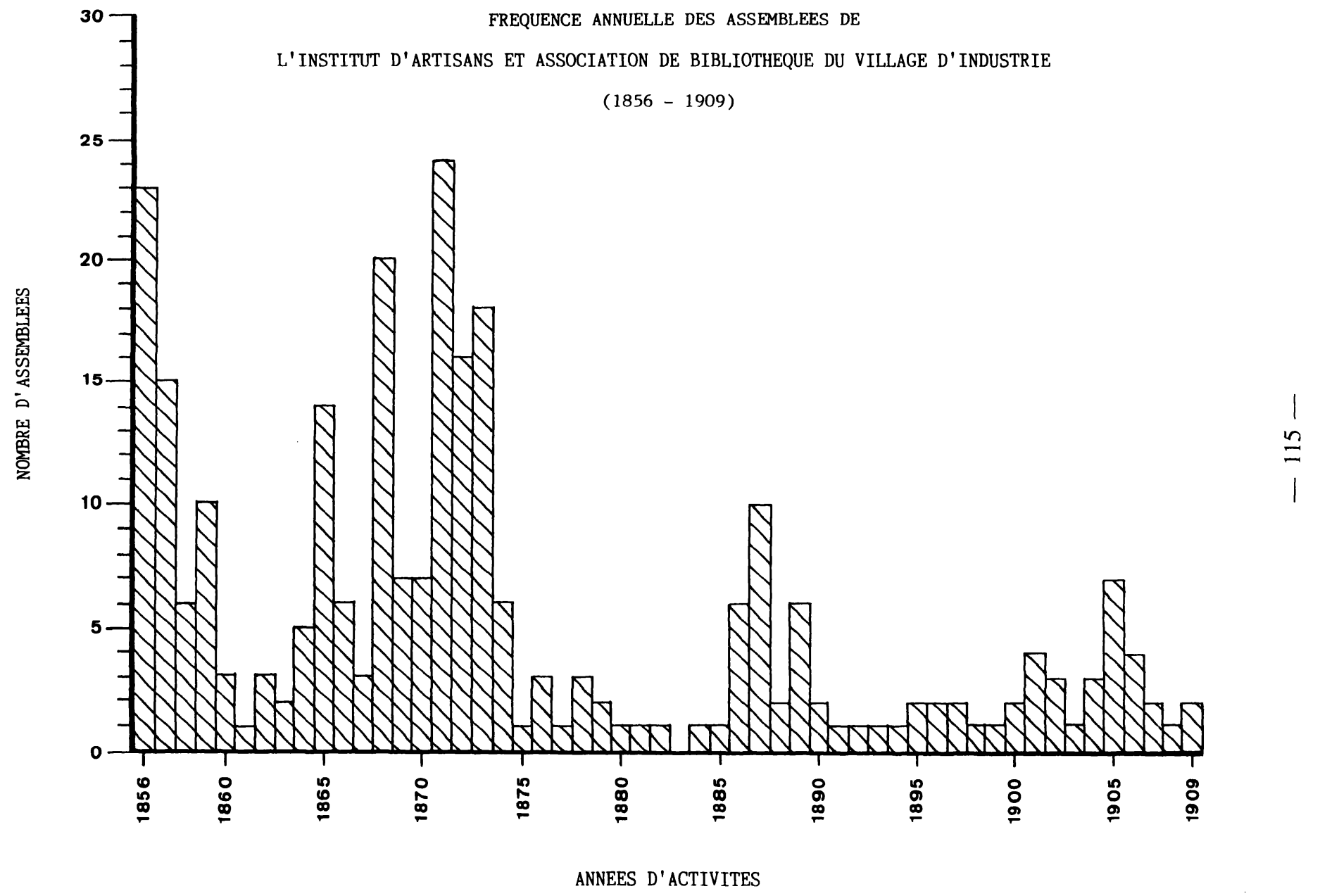

